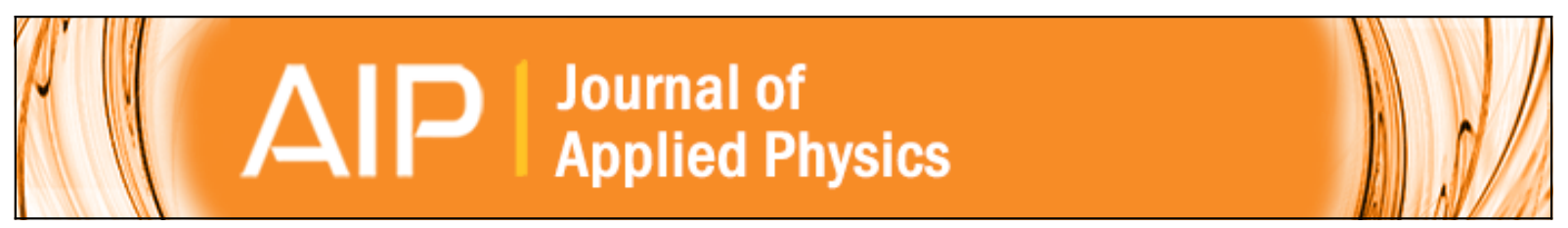

Photopolymerizable glasses incorporating high refractive index species and ionic liquid: A comparative study

A. V. Velasco, M. P. Hernández-Garay, M. L. Calvo, P. Cheben, and F. del Monte

Citation: Journal of Applied Physics 109, 053106 (2011); doi: 10.1063/1.3553840

View online: http://dx.doi.org/10.1063/1.3553840

View Table of Contents: http://scitation.aip.org/content/aip/journal/jap/109/5?ver=pdfcov

Published by the AIP Publishing

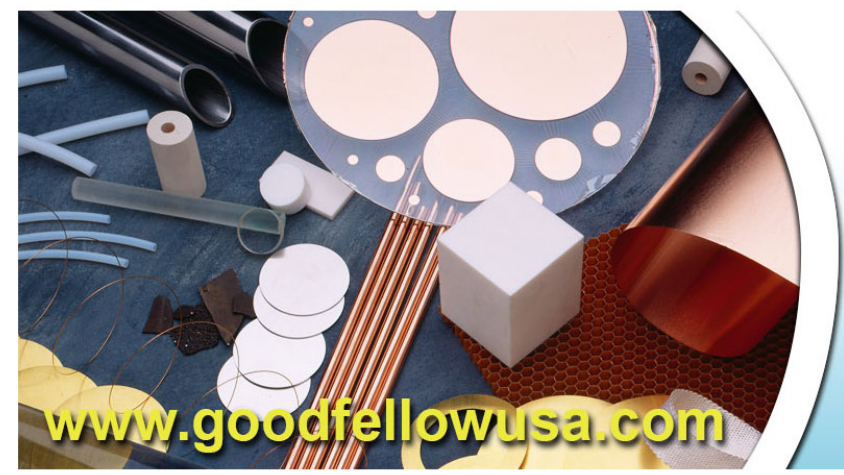

BDOIFEl|OII

metals • ceramics $\cdot$ polymers composites $\bullet$ compounds $\bullet$ glasses

Save $5 \% \cdot$ Buy online 70,000 products $\cdot$ Fast shipping 


\title{
Photopolymerizable glasses incorporating high refractive index species and ionic liquid: A comparative study
}

\author{
A. V. Velasco, ${ }^{1, a)}$ M. P. Hernández-Garay, ${ }^{1}$ M. L. Calvo, ${ }^{1}$ P. Cheben, ${ }^{2}$ and F. del Monte ${ }^{3}$ \\ ${ }^{1}$ Department of Optics, Faculty of Physics, Complutense University of Madrid, 28040 Madrid, Spain \\ ${ }^{2}$ Institute for Microstructural Sciences, National Research Council Canada, Ottawa, Ontario KIA OR6, \\ Canada \\ ${ }^{3}$ Institute for Materials Science, Spanish Research Council, 28049 Cantoblanco, Spain
}

(Received 10 November 2010; accepted 6 January 2011; published online 8 March 2011)

\begin{abstract}
Three different holographic photomaterials belonging to the class of photopolymerizable glasses have been synthesized using sol-gel techniques, and characterized with the purpose of a comparative study. Their behavior is analyzed in terms of achieved refractive index modulation, dark diffusion mechanism, diffraction efficiency and optical quality; in order to determine their suitability for different holographic applications. ㅇ 2011 American Institute of Physics. [doi:10.1063/1.3553840]
\end{abstract}

\section{INTRODUCTION}

The requirements of synthesis, characterization and development of recording materials exhibiting high dynamic range, high photosensitivity, and excellent optical quality, is still a challenging achievement in the field of photomaterials $^{1,2}$ and within the holographic data storage techniques. ${ }^{3-7}$ The fulfillment of these requirements shown by photopolymerizable materials has generated an important focus of attention on this family of holographic composites. ${ }^{8-10}$ As a result of the investigation in these materials, the so named photopolymerizable glasses developed with sol-gel techniques were first introduced in $1996 .{ }^{11}$

These hybrid organic-inorganic photosensitive glasses incorporate monomeric and photoinitiator species in a silicate matrix. When exposed to an interference pattern at an appropriate wavelength, the photoinitiator is activated and the polymerization of the free monomers takes place. The size increase of the resulting polymeric chains eventually hinders their diffusion within the glass matrix, thus making this distribution permanent after the recording. Additionally, after the polymerization, in regions of the interference pattern exposed to a higher light intensity distribution, the monomer concentration is depleted, resulting in a diffusion of the remaining monomer from the darker fringes to the illuminated ones.

As a result of this combined process of polymerization and diffusion, refractive index differences $\Delta n$ up to $5.6 \times 10^{-3}$ between dark and illuminated regions of the interference pattern have been achieved. ${ }^{12}$

These results have been further improved by incorporating in the photopolymerizable glass High Refractive Index Species (HRIS). ${ }^{12,13}$ In order to minimize scattering, zirconium isopropoxide $\mathrm{Zr}\left(\mathrm{O}^{\mathrm{i}} \mathrm{Pr}\right)_{4}$ was introduced at a molecular level to act as the HRIS, using methacrylic acid as a chelating agent to slow its hydrolysis. With the addition of the HRIS, refractive index modulations $(\Delta n)$ up to $10^{-2}$ are achieved. The relation between the incorporation of $\mathrm{ZrO}_{2}$ nanoparticles to polymeric films and the resulting recording

${ }^{\text {a)} E l e c t r o n i c ~ m a i l: ~ a v i l l a f r @ p d i . u c m . e s ~}$ sensitivity and gelation point have also been demonstrated ${ }^{14}$ as well as the aforementioned $\Delta n$ increase.

In another aspect of the investigation in photomaterials, and given the hazardous nature of some of the involved components, such as the monomer and sol-gel precursors, the introduction of nonvolatile substances in the design, synthesis and applications, is having a primary interest for introducing sustainable chemical processes. In particular, Ionic liquids (ILs) have recently focused great attention, also as an alternative to solvents in chemical reactions. ${ }^{15,16} \mathrm{ILs}$ are organic salts having an ionic structure (hence the name) and which are liquid at ambient temperature and exhibit hydrophilic behavior. Moreover, although ILs may be hazardous for the environment in some cases (in particular in aquatic environments), their extreme low nonvolatility prevents the evaporation of toxic substances which is typical of other solvents. Additionally, ionic liquids have shown to improve polymerization rates ${ }^{17-19}$ an advantage which has already been exploited in holographic materials based on photopolymers. ${ }^{20}$ In the preliminary studies done by Lin et al., the incorporation of IL to a photosensitive polymerizable material resulted in an increase of the sensitivity, spatial resolution and refractive index modulation. The strong diffusion mechanism which follows the light exposure, enhanced by the presence of IL, allowed to reach diffraction efficiencies up to $34 \%$ for a thin hologram (Raman-Nath regime, ${ }^{21} 10$ $\mu \mathrm{m}$ grating thickness). Holographic materials incorporating IL have also been used in the development of optical diffus$\mathrm{ers}^{22}$ as the concentration of IL can be varied to regulate their light diffusion property.

In this paper, and encouraged by the previous mentioned results, we present a comparative study among three alternative formula for the composition of a photopolymerizable glass: (1) the formula that contains HRIS, already studied by us, ${ }^{23-26}$ (2) incorporation of IL to the previous formula, and (3) exclusion of the HRIS in the composition while maintaining the IL as a component. A comparative study of the various physical performances obtained is presented.

The paper is organized as follows: Section II presents the results obtained with the photopolymerizable glass containing the HRIS. In particular, the angular selectivity and 
refractive index modulation are studied along with an analysis of the structure of the holographic gratings with Scanning Electron Microscope (SEM). The dark diffusion mechanism is also discussed. Section III is dedicated to the results obtained for the new formula of the photopolymerizable glass incorporating IL. In Sec. IV we present the results for the new formula incorporating both the HRIS and the IL and with studies that provides comparative analysis with the results presented in previous sections. Discussions and conclusions are presented in Sec. V.

\section{PHOTOPOLYMERIZABLE GLASS INCORPORATING HIGH REFRACTIVE INDEX SPECIES}

\section{A. Synthesis}

In previous publications, ${ }^{13}$ we have disclosed both the synthesis and the characterization analysis of the photopolymerizable glass incorporating HRIS. As an antecedent, this material has already shown strong performances, reaching $\Delta n$ of up to $10^{-2}$ and diffraction efficiencies close to $100 \%$ with a good optical quality. In particular, the applications for the implementation of transmission volume phase holographic gratings (VPHG) with the aim to operate as holographic optical elements (HOE) for various purposes and experiments, have demonstrated their flexibility for maintaining the high performance under a wide range of spatial frequencies and laser sources conditions for the recording and readout. ${ }^{23-26}$

However, in order to perform the comparative study with the photopolymerizable glasses incorporating IL, these analyses were revisited by using in the subsequent characterization samples synthesized at the same time than those with $\mathrm{IL}$, and thus ensuring the same synthesis conditions for all the photopolymerizable glasses of the present study.

Photopolymerizable glass samples incorporating HRIS were synthesized according to the previously disclosed process $^{13}$ (also described in the Appendix), with thicknesses ranging from 18 to $84 \mu \mathrm{m}$. Gelation was performed with a thermal treatment at a controlled temperature of $40^{\circ} \mathrm{C}$ during a period of $14 \mathrm{hs}$. As expected, and following the already well established characteristics, photopolymerizable glass samples incorporating HRIS showed an excellent surface quality and homogeneity, with very low scattering.

\section{B. Grating recording and characterization}

Volume phase holographic gratings (VPHG) were recorded in the photopolymerizable glass samples by interference of two mutually coherent $s$-polarized beams of equal intensities using a Mach-Zehnder type interferometer illuminated with a solid state laser beam centered at a wavelength of $532 \mathrm{~nm}$ (Oxxius 532S). An output power of $50 \mathrm{~mW}$ was equally divided between the two beams, which were incident on the sample at an angle of $7.9^{\circ}$ from the sample normal. The resulting interference fringes had a spatial frequency of 500 lines $/ \mathrm{mm}$. These conditions correspond to the Bragg diffraction regime.

The gratings were recorded shortly after the synthesis of the photopolymerizable glasses, with storage times prior to the recordings of up to $8 \mathrm{hs}$.
The evolution of the grating formation was monitored during the recording by a nonactinic probe beam from a He-Ne laser at $632.8 \mathrm{~nm}$ with an output power of $0.5 \mathrm{~mW}$ (Newport ULM-TILT). Angular selectivity of the recorded gratings was monitored by measuring the first Bragg diffracted order with the same $\mathrm{He}-\mathrm{Ne}$ laser and the sample placed on a high-precision rotation platform. $\Delta n$ values were obtained by fitting the resulting angular selectivity curve to the theoretical expression derived from Kogelnik's scalar first-order approximation for light diffraction by dielectric volume holographic gratings ${ }^{10}$ :

$$
\Delta n=2 n_{0} \operatorname{Cos}\left(\vartheta_{0}\right) \operatorname{Sin}\left(\vartheta_{0}\right) T \frac{\operatorname{ArcSin}(\sqrt{\eta})}{\sqrt{\pi^{2}-(\operatorname{ArcSin}(\sqrt{\eta}))^{2}}},
$$

where $n_{o}$ is the refractive index of the photomaterial prior to modulation; $\theta_{0}$ is the Bragg angle; $T$ is the sample thickness; and $\eta$ is the diffraction efficiency. The latter was calculated as $\eta=P_{-1} / P_{0}$, where $P_{-1}$ is the power in the -1 st diffraction order and $P_{0}$ is the power in the probe beam incident on the sample.

\section{Grating refractive index modulation and diffraction efficiency}

Figure 1 shows the obtained $\Delta n$ values for six different volume holographic gratings implemented in photopolymerizable glass samples containing HRIS, with a spatial frequency of 500 lines $/ \mathrm{mm}$. As mentioned before, note that refractive index modulations up to $1.0 \times 10^{-2}$ were achieved in earlier experiments with the same formula. ${ }^{13}$

Photopolymerizable glasses incorporating HRIS show a very good repeatability for refractive index modulation values among gratings implemented within the same synthesis procedure, and reaching, in this case, $\Delta n=8.7 \times 10^{-3}$.

$\Delta n$ is observed to remain constant in average versus the sample thickness. The high achieved values are a consequence of the efficient diffusion mechanism of the HRIS, as in the recording process the HRIS migrates from the dark fringes of the recording interference pattern to the

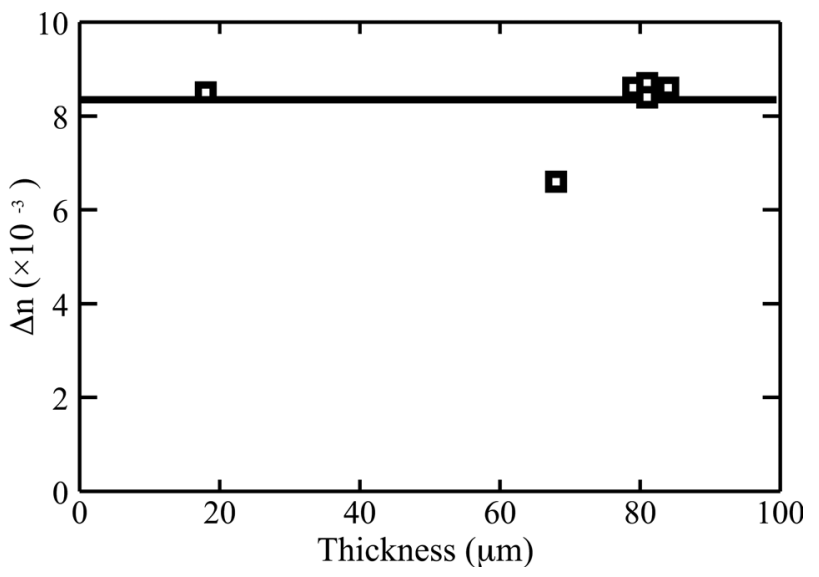

FIG. 1. Refractive index modulation vs holographic grating thickness in a photopolymerizable glass with HRIS. Spatial frequency 500 lines/mm. Volume gratings recorded with a solid-state laser at $532 \mathrm{~nm}$ and read with a He-Ne laser at $632 \mathrm{~nm}$ in photopolymerizable glasses incorporating HRIS. Straight line: $\Delta n$ average value. 
illuminated regions, thereby increasing the refractive index in the latter. ${ }^{13}$

Within the mentioned $\Delta n$ limits, diffraction efficiencies close to $100 \%$ can readily be achieved, as well as overmodulation regimes, as shown in Fig. 2.

\section{Dark diffusion mechanism}

After completion of the holographic exposure at the time of recording a diffraction grating, $\Delta n$ evolves under dark conditions. As a result of the polymerization of the monomeric species in the illuminated regions, the distribution of the monomer inside the glass matrix is not uniform, thus generating migrations of the monomer from the low refractive index regions to the high refractive index ones. The migrated monomer species may also become part of the polymer chains. This monomer diffusion process, which takes place in the absence of additional photon flux, is known as "dark diffusion", and stops when the composition reaches its chemical equilibrium.

In the case of the photopolymerizable glass with HRIS, the temporal evolution of $\Delta n$ after the holographic recording can be modeled using the weighted sum of two exponential functions ${ }^{20}$ :

$$
\Delta n(t)=C_{M}\left[1-\exp \left(-t / \tau_{M}\right)\right]+C_{Z r}\left[1-\exp \left(-t / \tau_{Z r}\right)\right]
$$

The two exponentials account for the contribution to $\Delta n$ temporal evolution of the monomer and the Zr-based HRIS, respectively, with diffusion time constants $\tau_{M}<\tau_{Z r}$. The exponential weight factors, $C_{M}$ and $C_{Z r}$, account for the contribution of monomer and HRIS components, respectively, to the overall refractive index modulation. Figure 3 shows an example of the fitting of an experimental recording of $\Delta n$ temporal evolution to Eq. (2). It is appreciated the excellent fitting with the experimental results, and the low noise of the curve.

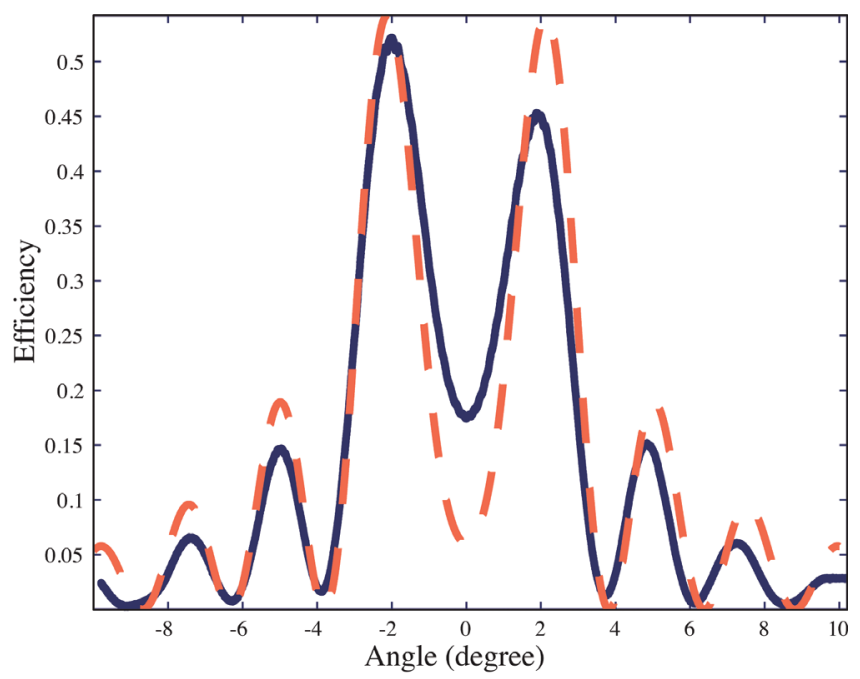

FIG. 2. (Color online) Experimental results (solid) and theoretical fitting (dashed) of the angular selectivity of an overmodulated volume grating recorded in photopolymerizable glass incorporating HRIS. Sample thickness is $80 \mu \mathrm{m}$ in the measured region. Refractive index modulation $\Delta n=8.6 \times 10^{-3}$ for a 500 lines $/ \mathrm{mm}$ grating recorded with a solid-state laser at $532 \mathrm{~nm}$ and read with a nonactinic He-Ne laser at $632 \mathrm{~nm}$.

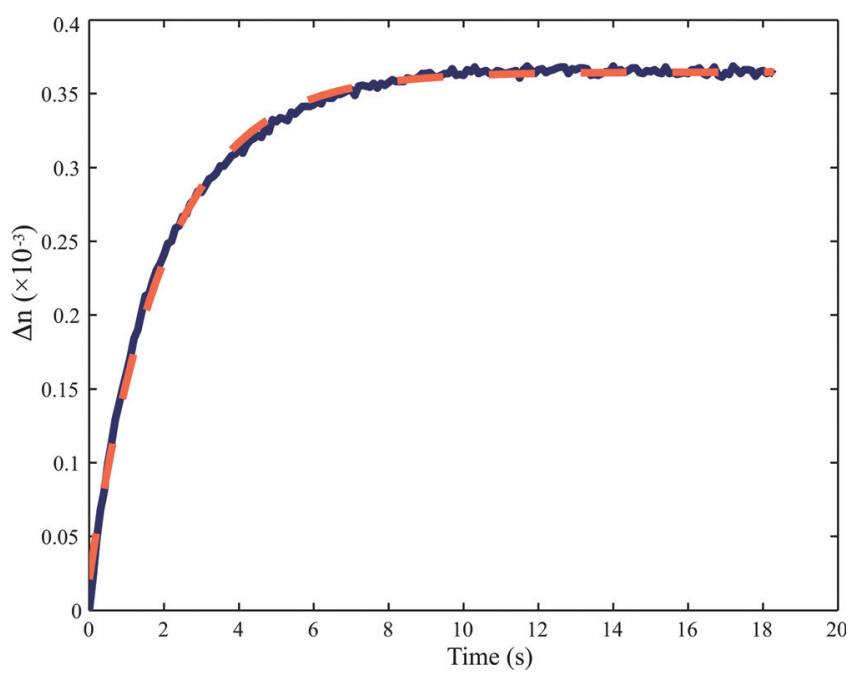

FIG. 3. (Color online) Experimental measurement (solid) and theoretical fitting (dash) of dark diffusion after a $2 \mathrm{~s}$ recording pulse at $532 \mathrm{~nm}$. The theoretical fitting corresponds to Eq. (2). Grating spatial frequency 500 lines $/ \mathrm{mm}$. $\Delta n$ was measured with a He-Ne laser at $632 \mathrm{~nm}$. Sample thickness $79 \mu \mathrm{m}$.

For a particular photopolymerizable glass sample, the four parameters that describe dark diffusion after a recording pulse $\left(C_{M}, \tau_{M}, C_{Z r}, \tau_{Z r}\right)$ vary depending on the number of recording pulses already applied to the material, as shown in Fig. 4.

After each pulse, two distinct diffusion processes appear: a fast diffusion associated to the monomeric species, with diffusion time constants on the order of $1 \mathrm{~s}$; and a slower diffusion associated with the HRIS, with diffusion time constants ranging from 2 to 6 seconds. The contribution of HRIS to the refractive index modulation is greater than the one of the monomeric species, with $C_{Z r}>C_{M}$ for all cases. Also, as the number of pulses increases, the presence of polymeric chains in the glass matrix makes high-index species diffusion more difficult, resulting in larger diffusion time constants.

Figure 5 shows the ranges of the diffusion time constant among recording pulses applied to each particular grating, for four different samples of photopolymerizable glasses with HRIS. In all the gratings, the two-exponential behavior and its associated values are consistent with the described results.

The light scattering in the grating samples was analyzed in previous papers ${ }^{13}$ and in which it was demonstrated the very low scattering level and the high quality of the diffracted beam profile.

\section{PHOTOPOLYMERIZABLE GLASS INCORPORATING IONIC LIQUID: A COMPOSITION WITHOUT HRIS}

\section{A. Synthesis}

Photopolymerizable glasses with two different IL concentrations (6\% and $4 \%$ of the total mass, respectively) were synthesized following a process similar to that of the glasses with HRIS (see the Appendix). The IL used in our composition was 1-butyl-3-methylimidazolium tetrafluoroborate ([bmim]BF4), from SIGMA-Aldrich. After casting the solgel solution, the samples were heated at $40^{\circ} \mathrm{C}$ during sixty hours up to the complete gelation of the photopolymerizable 

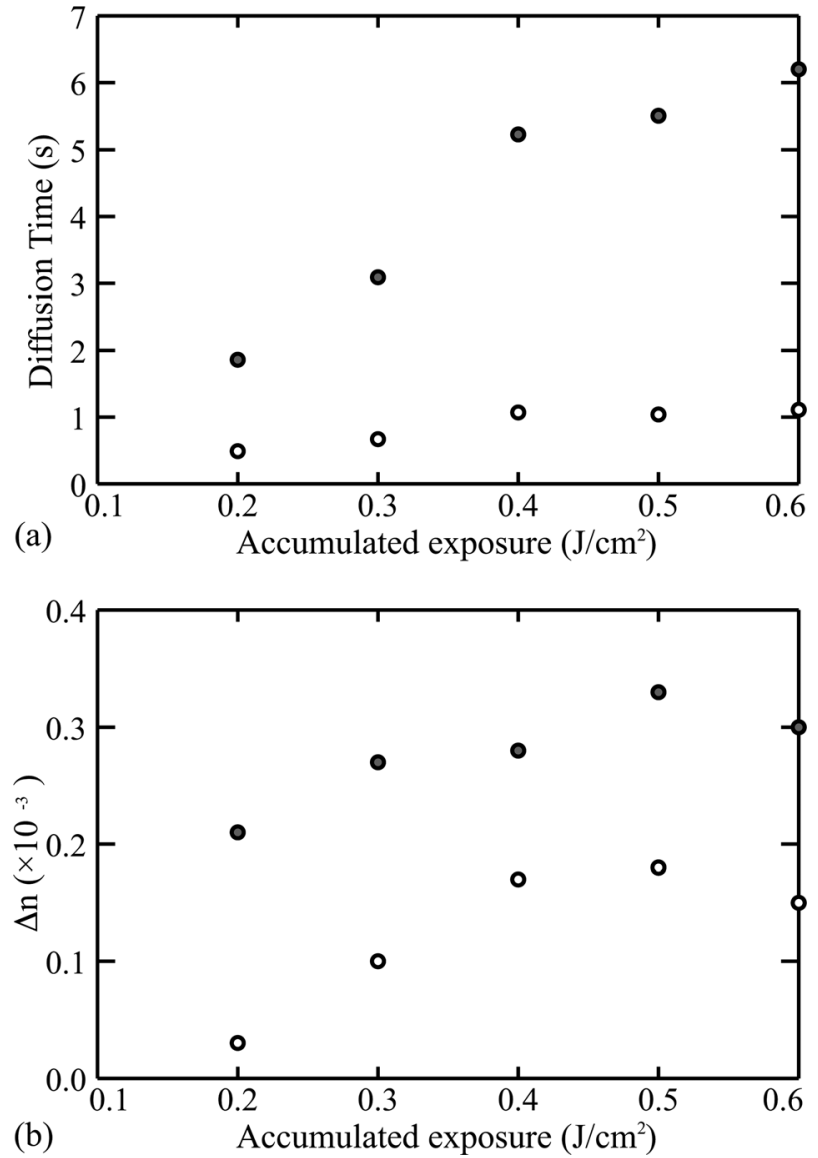

FIG. 4. (a) Diffusion time constants $\tau_{M}$ (open circles) and $\tau_{Z r}$ (solid circles); and (b) refractive index modulations $C_{M}$ (open circles), and $C_{Z r}$ (solid circles) for fitting to Eq. (2), as a function of accumulated exposure during the recording process. An $81 \mu \mathrm{m}$ thick sample incorporating HRIS. The duration of each recording pulse is $2 \mathrm{~s}$. The first pulse is not shown as it did not yield a measurable refractive index modulation. Maximum value of $\Delta n$ achieved is $8.6 \times 10^{-2}$ for a 500 lines $/ \mathrm{mm}$ grating, recorded with a solid state laser at $532 \mathrm{~nm}$ and measured with a He-Ne laser at $632 \mathrm{~nm}$.

glass samples. Samples were prepared with thicknesses ranging from 45 to $165 \mu \mathrm{m}$. At a first visual inspection, samples incorporating IL showed a good surface optical quality prior to the recording of VPHGs.

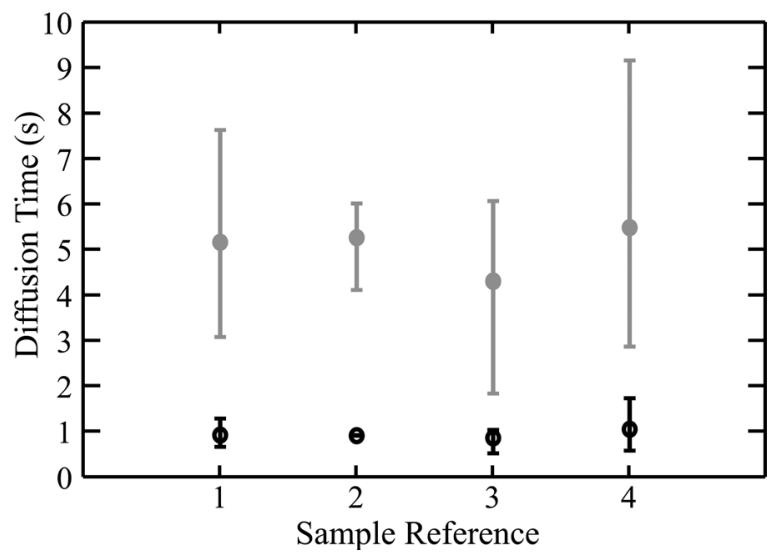

FIG. 5. Diffusion time constants $\tau_{M}$ (open circle) and $\tau_{Z r}$ (solid circle) for fitting to Eq. (2) of four samples of photopolymerizable glass incorporating HRIS. Sample 1: thickness $84 \mu \mathrm{m}, \Delta n=8.6 \times 10^{-3}$. Sample 2: thickness $79 \mu \mathrm{m}, \Delta n=8.6 \times 10^{-3}$. Sample 3: thickness $81 \mu \mathrm{m}, \Delta n=8.7 \times 10^{-3}$. Sample 4: thickness $81 \mu \mathrm{m}, \Delta n=8.5 \times 10^{-3} .500$ lines $/ \mathrm{mm}$ VPHG were recorded with a solid state laser at $532 \mathrm{~nm}$ and measured with a He-Ne laser beam at $632 \mathrm{~nm}$.

\section{B. Grating recording and characterization}

Following an identical experimental procedure as in Sec. II B., Bragg gratings of spatial frequency 500 lines $/ \mathrm{mm}$ were recorded in the samples.

\section{Grating refractive index modulation and diffraction efficiency}

The achieved refractive index modulation for gratings with 500 lines $/ \mathrm{mm}$ recorded in photopolymerizable glasses incorporating IL are shown in Fig. 6. The maximum refractive index modulation $\Delta n=5 \times 10^{-3}$, is found for a VPHG implemented in a glass sample synthesized with $6 \%$ of IL. The average value of $\Delta n$ obtained with this concentration $\left(3.35 \times 10^{-3}\right)$ is also slightly higher than the one achieved with the $4 \%$ concentration $\left(2.95 \times 10^{-3}\right)$. However, the VPHGs recorded in the material with the higher concentration of IL (6\%) also show a larger variability, with a higher range of resulting $\Delta n$ values (from $2 \times 10^{-3}$ for a $45 \mu \mathrm{m}$ thickness sample to $5 \times 10^{-3}$ for a $110 \mu \mathrm{m}$ thickness sample). Therefore, for the sake of a comparative study, the $4 \%$ concentration samples exhibit a more stable behavior.

In all studied cases, VPHGs recorded in photopolymerizable glasses incorporating IL show lower $\Delta n$ values than those disclosed in Sec. II for the photopolymerizable glasses incorporating HRIS. This is expected since HRIS is known to contribute significantly to the overall refractive index modulation. These results are also within the range usually found for the original formula of photopolymerizable glasses which do not incorporate neither IL nor HRIS. ${ }^{11}$ Thus, no relevant increase in $\Delta n$ results with the incorporation of IL in the composition, although other properties of the material related to dark diffusion mechanism and light scattering are indeed affected as shown in Secs. III D and III E.

Notwithstanding reduce $\Delta n$ values compared to those obtained for VPHGs recorded in photopolymerizable glasses incorporating only HRIS, overmodulation regimes can also be reached. An example of overmodulated VPHG is shown

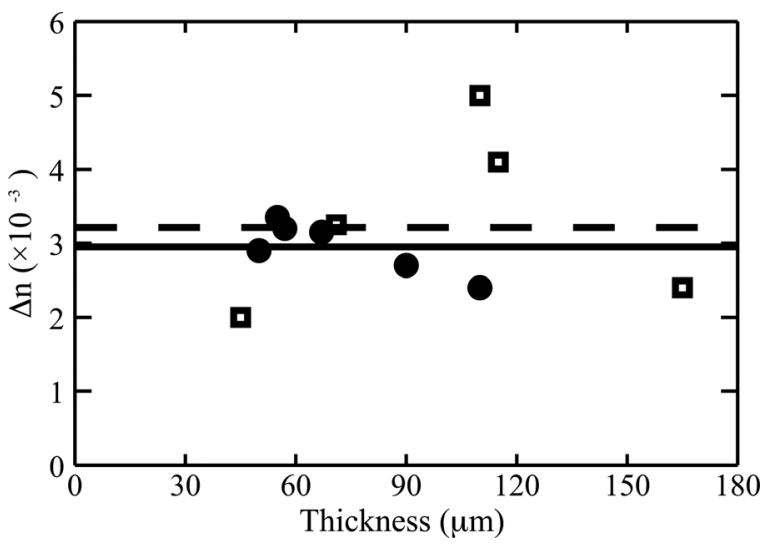

FIG. 6. Refractive index modulation vs holographic grating thickness. Spatial frequency 500 lines/mm. VPHG recorded with a solid-state laser at $532 \mathrm{~nm}$ and read with a nonactinic He-Ne laser beam at $632 \mathrm{~nm}$ in photopolymerizable glasses incorporating IL. Solid circle: $6 \%$ IL ( $\Delta n$ average value: dashed line). Open square: $4 \%$ IL ( $\Delta n$ average value: straight line). 


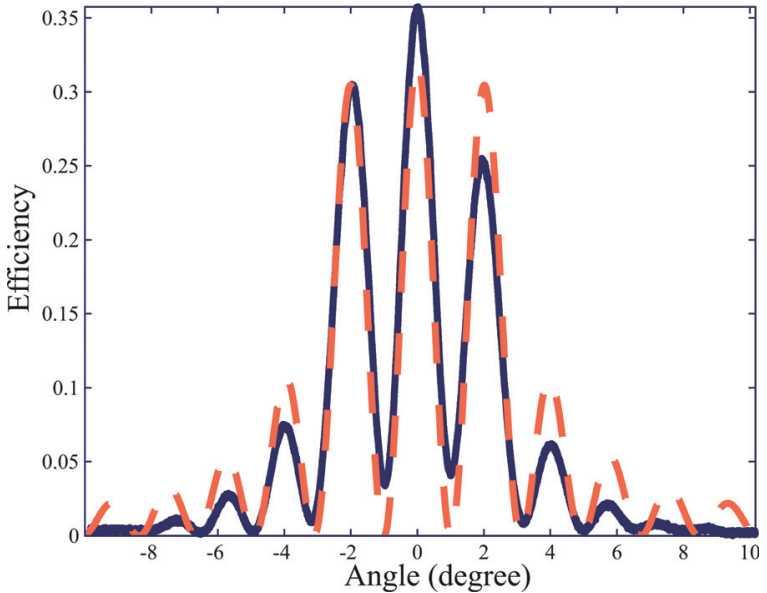

FIG. 7. (Color online) Experimental results (solid) and theoretical fitting (dashed) of the angular selectivity of an overmodulated volume grating recorded in photopolymerizable glass incorporating 6\% IL. Sample thickness is $100 \mu \mathrm{m}$ in the measured region. Refractive index modulation $\Delta \mathrm{n}=5 \times 10^{-3}$ for a 500 lines/mm grating recorded with a solid-state laser beam at $532 \mathrm{~nm}$ and read with a nonactinic He-Ne laser at $632 \mathrm{~nm}$.

in Fig. 7 by means of its angular selectivity, which shows three strong peaks near the Bragg angle.

Diffraction efficiencies close to $100 \%$ can also be reached with an appropriate exposure by preventing the overmodulation of the VPHGs.

\section{Dark diffusion mechanism}

Unlike the case of photopolymerizable glass incorporating HRIS, the dark diffusion mechanism shown by glass samples incorporating IL can be fitted to a single exponential function, Eq. (3). In this case, obviously only the contribution of the monomer diffusion (first term to the rhs of Eq. (2)) operates. A good precision for this fitting can be observed in Fig. 8, although, as the $\Delta n$ obtained is lower than in Fig. 3, the curve presents a lower signal-to-noise ratio. As a consequence, the ripple in Fig. 8 appears to be

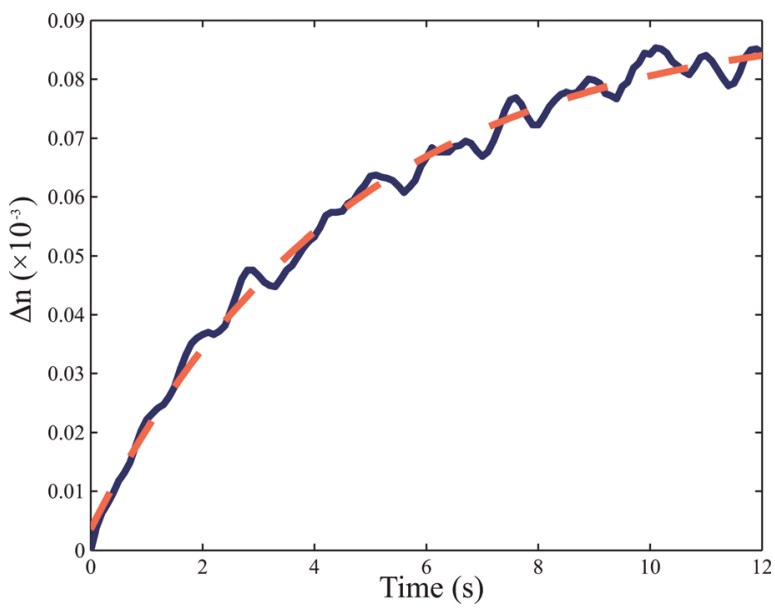

FIG. 8. (Color online) Experimental measurement (solid) and theoretical fitting (dashed) of dark diffusion after a 2 second recording pulse at $532 \mathrm{~nm}$ for a photopolymerizable glass incorporating IL. The theoretical fitting corresponds to Eq. (2). Grating spatial frequency 500 lines $/ \mathrm{mm}$. $\Delta n$ was measured with a nonactinic He-Ne laser at $632 \mathrm{~nm}$. Sample thickness is $110 \mu \mathrm{m}$.
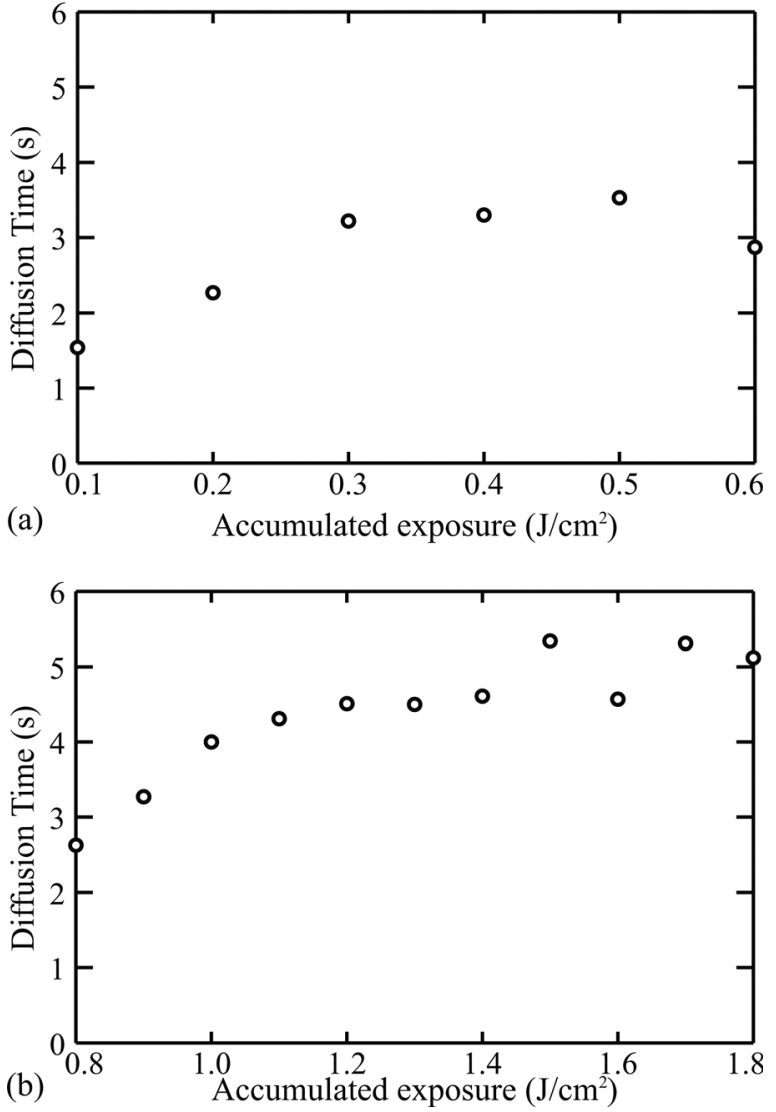

FIG. 9. (a) Diffusion time constants $\tau_{M}$ for fitting to Eq. (3) as a function of accumulated exposure during the recording process. Thickness $110 \mu \mathrm{m}$. IL concentration $6 \%$. (b) Thickness $55 \mu \mathrm{m}$. IL concentration 4\%. The duration of each recording pulse is $2 \mathrm{~s}$. Pulses not shown did not yield a significant refractive index modulation. Final $\Delta n$ achieved are $4.85 \times 10^{-3}$ (a) and $3.5 \times 10^{-3}$ (b) for a 500 lines/mm grating, recorded with a solid state laser at $532 \mathrm{~nm}$ and measured with a nonactinic He-Ne laser at $632 \mathrm{~nm}$.

greater than in Fig. 3, as the amplitude of the curve is smaller, although the absolute amount of noise is similar in both cases.

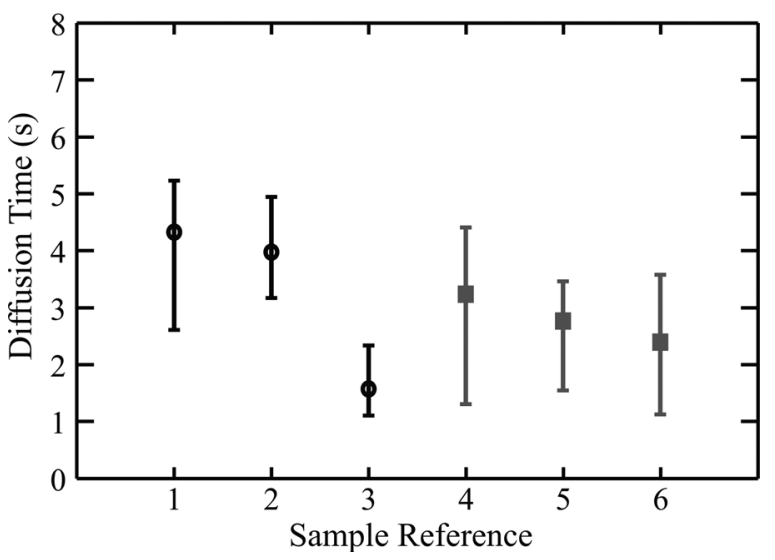

FIG. 10. Diffusion time constants $\tau_{M}$ for fitting to Eq. (3) of three samples of photopolymerizable glass incorporating $4 \%$ IL (open circle) and three samples incorporating 6\% IL (solid square). Sample 1: thickness $55 \mu \mathrm{m}$, $\Delta n=3.35 \times 10^{-3}$. Sample 2: thickness $50 \mu \mathrm{m}, \Delta n=2.9 \times 10^{-3}$. Sample 3: thickness $57 \mu \mathrm{m}, \Delta n=3.2 \times 10^{-3}$. Sample 4: thickness $110 \mu \mathrm{m}$, $\Delta n=4.85 \times 10^{-3}$. Sample 5: thickness $115 \mu \mathrm{m}, \Delta n=4.1 \times 10^{-3}$. Sample 6: thickness $165 \mu \mathrm{m}, \Delta n=2.9 \times 10^{-3} .500$ lines/mm VPHG were recorded with a solid state laser at $532 \mathrm{~nm}$ and measured with a nonactinic $\mathrm{He}-\mathrm{Ne}$ laser beam at $632 \mathrm{~nm}$. 
(a)

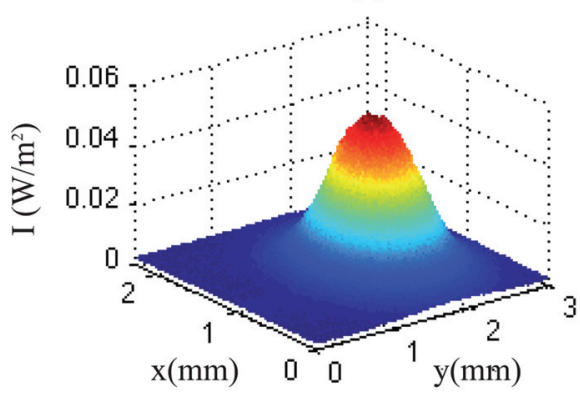

(c)

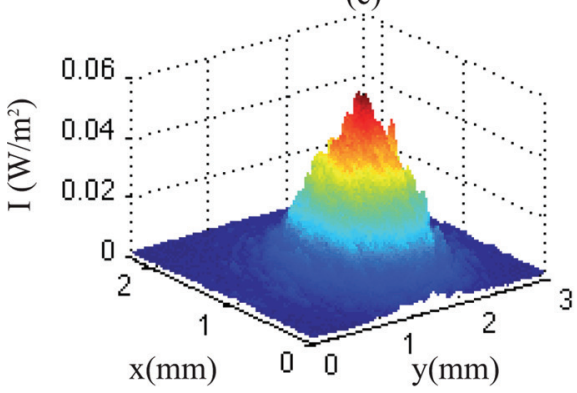

(b)

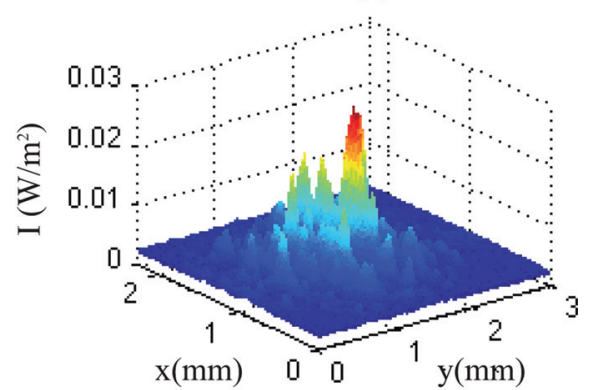

(d)

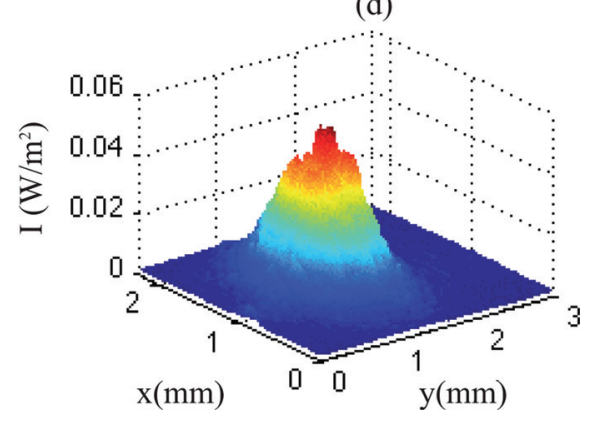

FIG. 11. (Color online) Incident beam (a), firstorder diffracted beam without surface scattering compensation (b), zero-th order diffracted beam with surface scattering compensation (c) and first order diffracted beam with surface scattering compensation (d), for a 500 lines $/ \mathrm{mm}$ grating implemented in a photopolymerizable glass incorporating a IL concentration of $6 \%$. Measurements obtained with a nonactinic $\mathrm{He}-\mathrm{Ne}$ laser at $632 \mathrm{~nm}$.

$$
\Delta n(t)=C_{M}\left[1-\exp \left(-t / \tau_{M}\right)\right]
$$

The absence of a second exponential term in the rhs of Eq. (3) confirms that the IL does not act as a photoactive element and it does not contribute in an independent manner to the refractive index modulation. However, as a direct consequence of the presence of the IL in the glass matrix, the diffusion time constants $\left(\tau_{M}\right)$ of the monomer dark diffusion present higher values, than those shown in Fig. 5. These values typically range between 1 and 5 seconds.

Figure 9 presents an example of the evolution of the diffusion time constant during a VPHG recording process. As in the results shown in Sec. II D, the diffusion time constant tends to increase with the temporal evolution of the recording process, as the presence of polymer chains inside the glass matrix hinder particle diffusion.

The aforementioned longer diffusion time constants compared to those obtained for the free monomer in the presence of HRIS, are common to all analyzed samples, of a total of six, that incorporate IL, as shown in Fig. 10.

\section{E. Light scattering}

The incorporation of IL to the photopolymerizable glass composition also has a direct impact on the light scattering and on the optical quality of the sample after the recording of the VPHG. Spherical cavities with typical diameters of 1-3 $\mu \mathrm{m}$ appear both on the surface and on the bulk of the glass matrix, increasing the light scattering and thus affecting the light diffracted intensity distribution. Further studies, performed by comparing the first-order diffracted beam before and after using a refractive index compensator (Norland Index Matching Liquid 150, $n=1.52$ ) to minimize the effect of surface irregularities, show that most of the light scattering originates on the surface of the samples, and can thus be readily compensated for. An example of this comparison study can be found in Fig. 11.

It should be noted that, although the light scattering increases due to the presence of IL, and it is undesirable for data storage applications, it can be nevertheless taken advantage for other applications such as optical diffusers, specially since the amount of light scattering introduced by the photopolymerizable glass can be controlled by varying the amount of IL included in the sample.

\section{F. SEM analysis}

Figure 12 presents a SEM image (obtained with Zeiss DSM-950 instrument $\{$ PossibleAd $\}$ ) of a $30 \mu \mathrm{m}$ thickness photopolymerizable glass sample incorporating, in this case, $7.6 \%$ IL for an enhanced observation of the phenomena associated

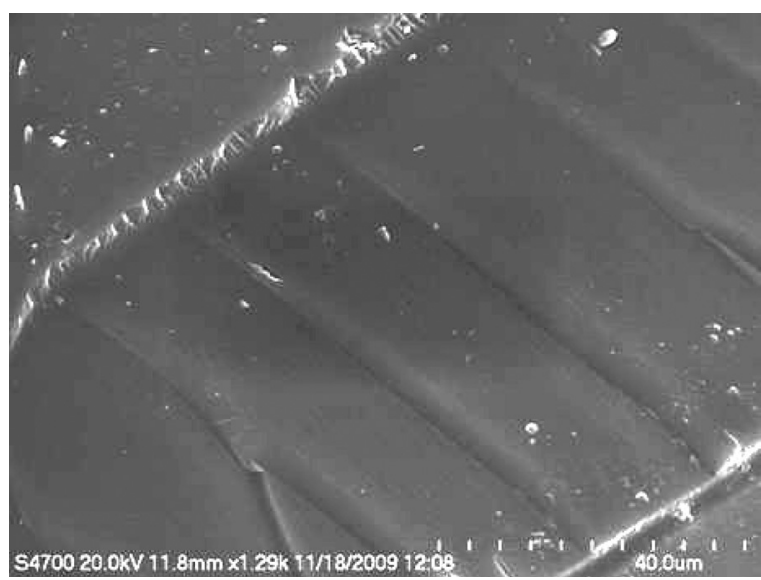

FIG. 12. SEM image of a VPHG implemented in a photopolymerizable glass incorporating IL. IL concentration is $7.6 \%$. Sample thickness is $30 \mu \mathrm{m}$ and grating period is $20 \mu \mathrm{m}$. 


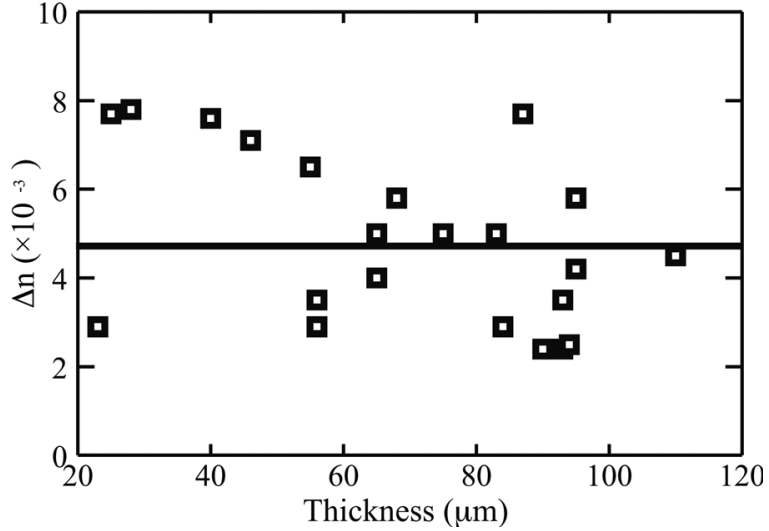

FIG. 13. Refractive index modulation vs holographic grating thickness. Spatial frequency 500 lines $/ \mathrm{mm}$. Volume gratings recorded with a solid-state laser at $532 \mathrm{~nm}$ and read with a nonactinic He-Ne laser at $632 \mathrm{~nm}$ in photopolymerizable glasses incorporating both HRIS and IL (concentration $3.2 \%)$. Straight line: $\Delta n$ average value.

to the incorporation of IL to the sample. The presence of a 50 lines/mm grating is apparent, as well as the generation of the aforementioned cavities, with diameters of up to $\sim 3 \mu \mathrm{m}$.

Although the period of the VPHG of the image $(\Lambda=20 \mu \mathrm{m})$ is greater than the size of the cavities, in a VPHG with a higher spatial frequency (for example, 500 lines $/ \mathrm{mm}$ and period $\Lambda=20 \mu \mathrm{m}$ ), the size of the cavities would be similar to the period of the VPHG, thus interfering with its refractive index pattern.

\section{PHOTOPOLYMERIZABLE GLASSES INCORPORATING BOTH HRIS AND IONIC LIQUID}

\section{A. Synthesis}

Photopolymerizable glasses incorporating both HRIS and IL were also synthesized following a similar process to those disclosed in Secs. II and III (see Appendix), obtaining samples ranging from 25 to $110 \mu \mathrm{m}$ thickness. Glass samples

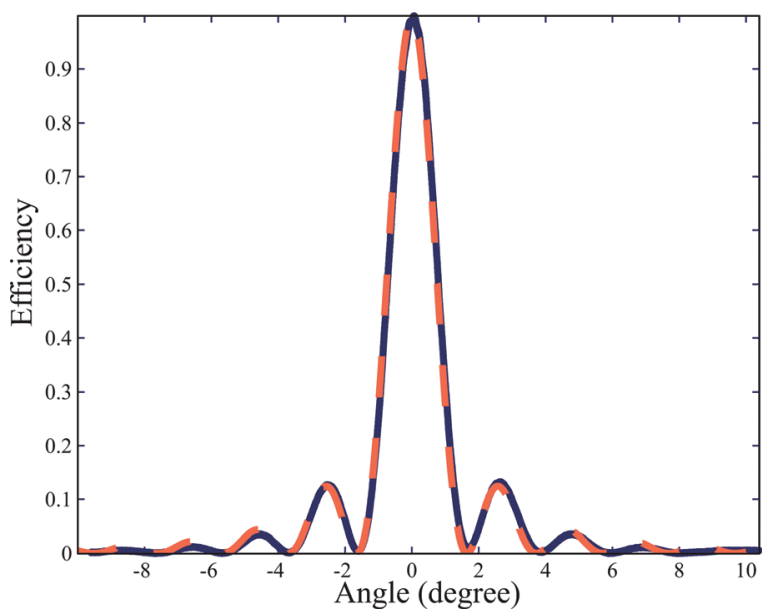

FIG. 14. (Color online) Experimental results (solid) and theoretical fitting (dashed) of the angular selectivity of VPHG recorded in a photopolymerizable glass incorporating HRIS and IL (concentration 3.2\%). Sample thickness is $93 \mu \mathrm{m}$ in the measured region. Refractive index modulation $\Delta n=3.5 \times 10^{-3}$ for a 500 lines $/ \mathrm{mm}$ grating recorded with a solid-state laser at $532 \mathrm{~nm}$ and read with a nonactinic He-Ne laser at $632 \mathrm{~nm}$.

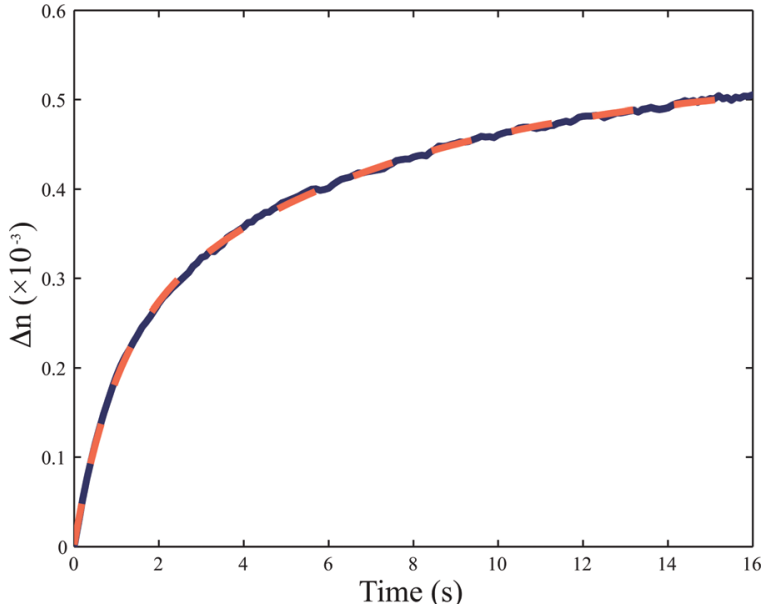

FIG. 15. (Color online) Experimental measurement (solid) and theoretical fitting (dashed) of dark diffusion after a 2 second recording pulse at $532 \mathrm{~nm}$ for a photopolymerizable glass incorporating both HRIS and IL (concentration $3.2 \%$ ). The theoretical fitting corresponds to Eq. (2). Grating spatial frequency 500 lines $/ \mathrm{mm}$. $\Delta n$ was measured with a nonactinic He-Ne laser at $632 \mathrm{~nm}$. Sample thickness is $110 \mu \mathrm{m}$.

exhibited a fast deterioration once gelated, presenting opaque areas on their surface. This phenomenon indicates instabilities in the coexistence of the IL and the HRIS within the glass matrix.
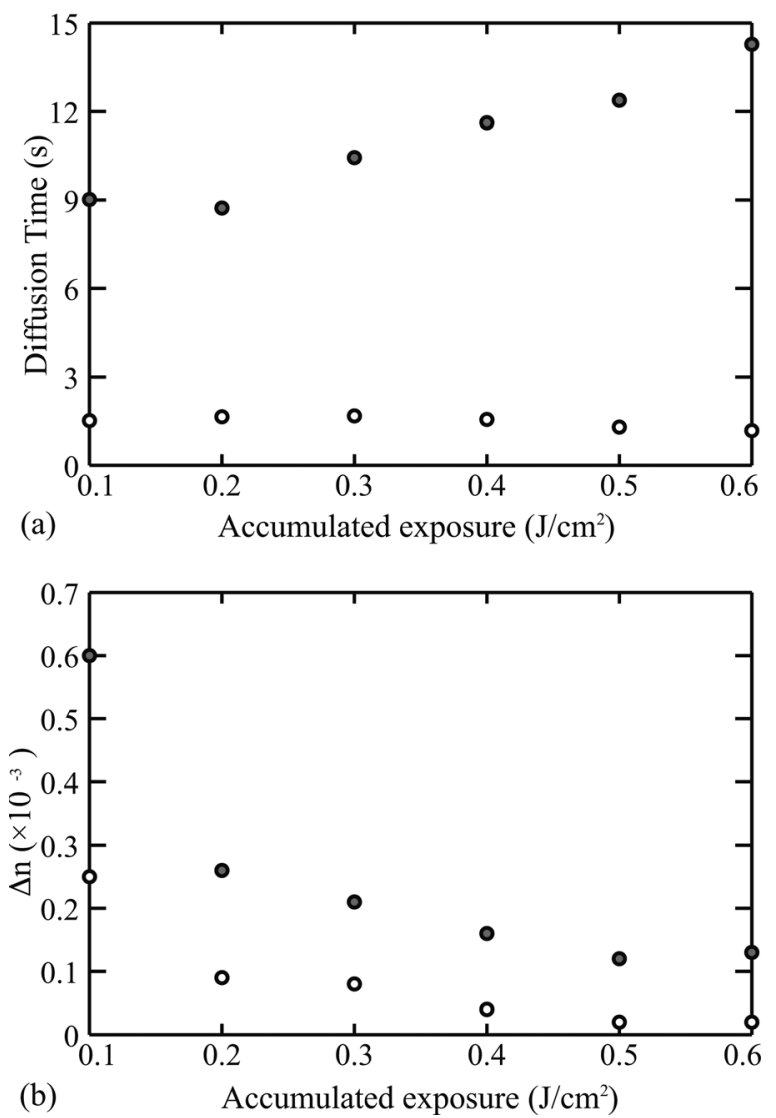

FIG. 16. (a) Diffusion time constants $\tau_{M}$ (open circle) and $\tau_{Z r}$ (solid circle); and (b) refractive index modulations $C_{M}$ (open circle), and $C_{Z r}$ (solid circle) for fitting to Eq. (2) as a function of accumulated exposure during the recording process, for a $94 \mu \mathrm{m}$ thick photopolymerizable glass sample incorporating both HRIS and IL (IL concentration: $3.2 \%$ ). The duration of each recording pulse is $2 \mathrm{~s}$. Final $\Delta n$ achieved is $2.5 \times 10^{-3}$ for a 500 lines $/ \mathrm{mm}$ grating, recorded with a solid state laser beam at $532 \mathrm{~nm}$ and measured with a nonactinic $\mathrm{He}-\mathrm{Ne}$ laser beam at $632 \mathrm{~nm}$. 


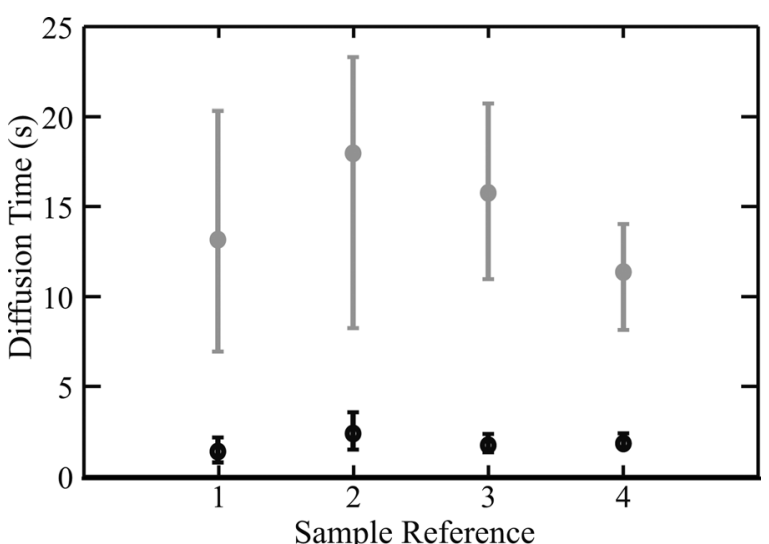

FIG. 17. Diffusion time constants $\tau_{M}$ (open circle) and $\tau_{Z r}$ (solid circle) for fitting to Eq. (2) of four samples of photopolymerizable glass incorporating HRIS and IL (concentration 3.2\%). Sample 1: thickness $28 \mu \mathrm{m}$, $\Delta n=7.8 \times 10^{-3}$. Sample 2: thickness $23 \mu \mathrm{m}, \Delta n=2.9 \times 10^{-3}$. Sample 3: thickness $93 \mu \mathrm{m}, \Delta n=3.5 \times 10^{-3}$. Sample 4: thickness $56 \mu \mathrm{m}$, $\Delta n=5.8 \times 10^{-3} .500$ lines $/ \mathrm{mm}$ VPHG were recorded with a solid state laser at $532 \mathrm{~nm}$ and measured with a nonactinic He-Ne laser at $632 \mathrm{~nm}$.

\section{B. Grating recording and characterization}

Bragg gratings of spatial frequency 500 lines/mm were recorded and characterized using the same method as disclosed in Sec. II B.

\section{Grating refractive index modulation and diffraction efficiency}

Figure 13 presents $\Delta n$ reached for a set of 500 lines $/ \mathrm{mm}$ gratings recorded in a photopolymerizable glass incorporating both HRIS and IL. Although values up to $\Delta n=7.8 \times 10^{-3}$ were reached, results showed a poor repeatability, with $\Delta n$ ranging from $7.8 \times 10^{-3}$ to $2.5 \times 10^{-3}$ under the same synthesis and recording conditions.

As a consequence of these results, although $\Delta n$ values close to those obtained with photopolymerizable glasses incor-

porating only HRIS can be obtained, the instability of the mixture of HRIS and IL (possibly due to influence on hydrolysis of $\mathrm{Zr}$ precursor) results in an increased uncertainty in $\Delta n$ achieved.

An example of an optimized angular selectivity of a VPHG recorded in a photopolymerizable glass incorporating both HRIS and IL is shown in Fig. 14, with a diffraction efficiency close to $100 \%$.

\section{Dark diffusion mechanism}

The measured dark diffusion of photopolymerizable glasses incorporating both IL and HRIS presents a good fit to Eq. (2). As in the case of glasses incorporating only HRIS, two diffusion processes with different kinetics can be distinguished. The faster diffusion component corresponds to the monomeric species, while the slower one accounts for the migration of the HRIS. Figure 15 shows an excellent fit to the model.

As we discussed in Sec. III, IL does not directly modify the $\Delta n$ value, but it does interact with the monomer and the HRIS inside the glass matrix, by increasing the diffusion time constants of the components. This effect can be seen in Fig. 16. In this case, HRIS shows a greater contribution to the $\Delta n$ than the monomer, both decreasing with the number of recording pulses.

Figure 17 shows the range of diffusion time constants for a variety of photopolymerizable glass samples incorporating both HRIS and IL. The diffusion times for the monomer and the HRIS are shown to be maintained for a longer period of time in the presence of IL, compared to the case of the material incorporating only HRIS (see Sec. II D).

\section{E. Light scattering}

The optical quality and light scattering properties of a photopolymerizable glass incorporating both HRIS and IL present a great variability among samples, as a consequence

(b)
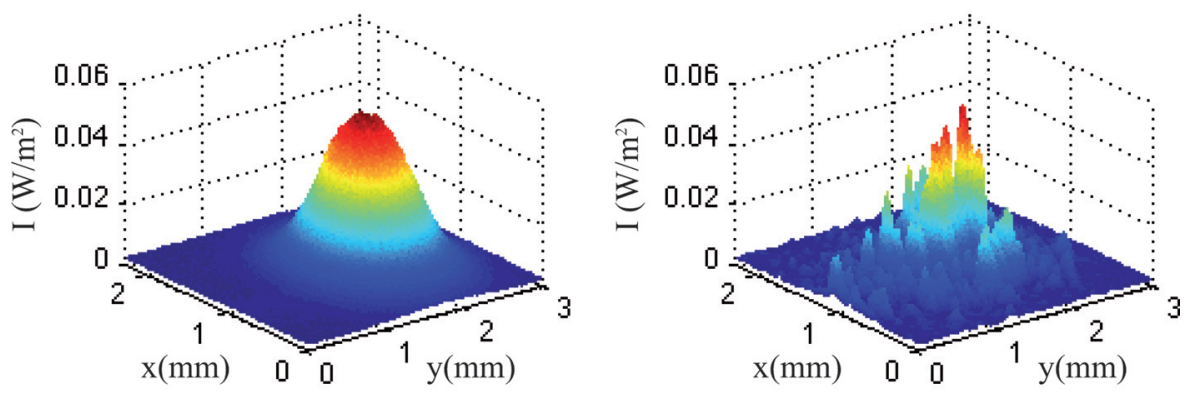

(c)

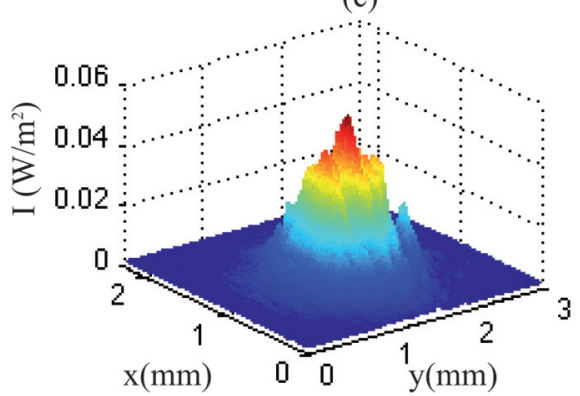

(d)

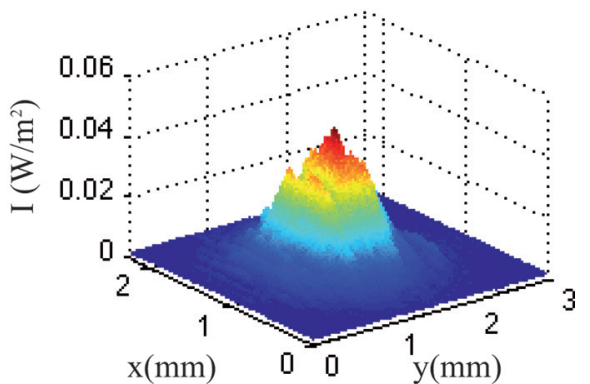

FIG. 18. (Color online) Incident beam (a), firstorder diffracted beam without surface scattering compensation (b), zero-th order diffracted beam with surface scattering compensation (c) and first order diffracted beam with surface scattering compensation (d) for a 500 lines $/ \mathrm{mm}$ grating implemented in a photopolymerizable glass incorporating HRIS and a IL concentration of $3.2 \%$. Measurements obtained with a $\mathrm{He}-\mathrm{Ne}$ laser at $632 \mathrm{~nm}$. 
(a)

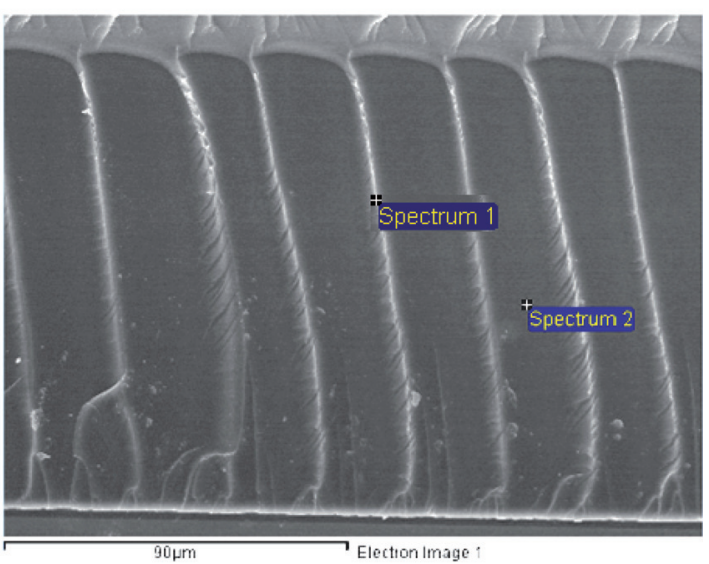

(b)

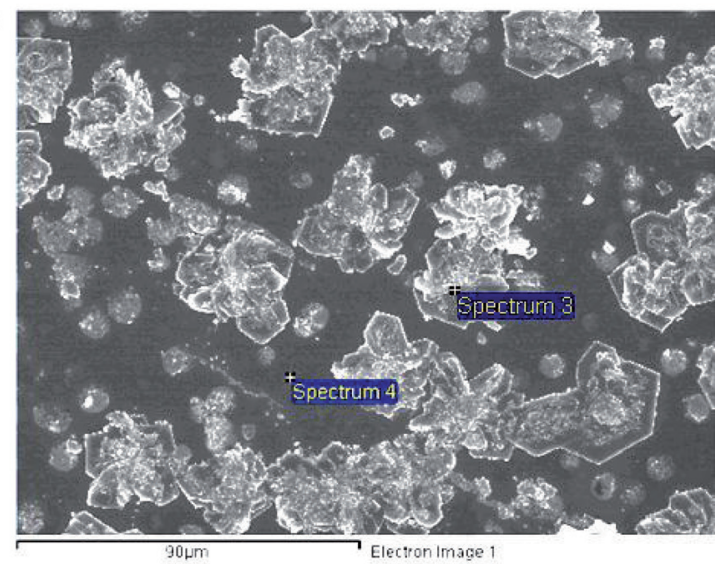

FIG. 19. (Color online) SEM images of the surface of a photopolymerizable glass incorporating both HRIS and IL (a), and of a volume grating formed in the glass matrix (b). Positions where spectral analysis was performed are also indicated.

of an apparently unstable coexistence of HRIS and IL inside the glass matrix. Samples prepared under similar conditions range from an acceptable optical quality to poor surfaces presenting certain irregularities and haze. An example is shown in Fig. 18.

\section{F. SEM analysis}

Figure 19 shows SEM images (obtained with a Zeiss DSM-950 instrument) in which the scattering aggregates are apparent. The percentage of zirconium concentration in different regions of a sample (see Table I) was determined by means of spectral analysis. Similar low amounts of zirconium were found in the dark and light fringes of a 50 lines/ $\mathrm{mm}$ VPHG implemented in the glass. The surface of the glass (a) showed a markedly increased zirconium concentration compared to the grating region (b), especially in areas where the aggregates were formed. The HRIS appear to have

TABLE I. Zirconium concentration of a photopolymerizable glass incorporating HRIS and IL.

\begin{tabular}{lc}
\hline \hline Position & Zr concentration (\%) \\
\hline Spectrum 1 & 2.59 \\
Spectrum 2 & 2.88 \\
Spectrum 3 & 20.26 \\
Spectrum 4 & 9.00 \\
\hline \hline
\end{tabular}

been expelled from the glass matrix, then reducing or even annulling its contribution to the $\Delta n$ of the VPHG. Obviously, the aggregations of HRIS on the surface of the photopolymerizable glass greatly reduces the optical quality of the affected samples.

\section{DISCUSSIONS AND CONCLUSIONS}

Three types of photopolymerizable glasses have been presented and systematically characterized in terms of refractive index modulation, angular selectivity, dark diffusion mechanism and light scattering. The photomaterials were synthesized following similar procedure previously implemented in other studies.

The photopolymerizable glass incorporating HRIS, already disclosed in previous publications, exhibits very high diffraction efficiency (near 100\%) and refractive index modulation (up to $\Delta n=1.0 \times 10^{-2}$ ), as well as a very good optical quality, the parameters relevant for optical data storage applications.

The photopolymerizable glass incorporating ionic liquid, disclosed here for the first time, exhibits a lower refractive index modulation (up to $\Delta n=5 \times 10^{-3}$ ), but still presents enough dynamic range to reach diffraction efficiencies close to $100 \%$ and overmodulation regimes. The presence of IL enhances the diffusion of the monomeric species.

Finally, the photopolymerizable glass incorporating both HRIS and IL, also disclosed here for the first time, show comparatively high refractive index modulations (up to $\Delta n=7.8 \times 10^{-3}$ ), although instabilities in the coexistence of HRIS and IL may result in precipitation of Zr-based components. This phenomenon implies a greater variability in achieved $\Delta n$ values and optical qualities. Diffusion time constants of both HRIS and monomer are increased by the presence of IL.

\section{APPENDIX: SAMPLES PREPARATION}

The chemical procedure for the synthesis of the photopolymerizable glass incorporating HRIS was reported in detail in Ref. 13 here we summarize the basic steps:

The silica sol is prepared by acid hydrolysis of glycidoxypropyltrimethoxysilane (GPTMS) and tetraethylorthosilicate (TEOS). After 10 mins of stirring, a solution of IRGAGURE-784 photosensitizer in POEA is added. After further 10 mins of stirring, a solution of zirconium isopropoxide isopropanol complex in methacrylic acid is added to the mixture. The resulting sol is filtered with a $0.2 \mu \mathrm{m}$ Millipore filter.

For the synthesis of the photopolymerizable glass incorporating IL we have used the analogous chemical formula but $\mathrm{ZrO}_{2}$ was not included, being replaced by the IL (namely, 1-Butyl-3 methylimidazolium tetrafluorborate, ALDRICH). Two particular weights of IL were applied for two types of samples: $4 \%$ and $6 \%$ of the total mass.

For the synthesis of the photopolymerizable glass incorporating HRIS and IL a similar procedure as the previous one was followed but the HRIS was incorporated before adding the IL (weights: 9.5\% HRIS and 3.2\% IL, respectively, of the total mass). 


\section{ACKNOWLEDGMENTS}

Financial support from the Spanish Ministry of Science and Innovation (MICINN) under grant TEC2008-04105 is acknowledged.

A.V.V. acknowledges a fellowship from MICINN and M.P.H.G. acknowledges a fellowship from the Spanish Ministry of External Affairs and CONACyT (Mexico).

We thank O. Martínez-Matos for helpful suggestions and discussions.

${ }^{1}$ M. L. Calvo, P. Cheben, Advances in Information Optics and Photonics, edited by Ari T. Friberg, R. Dändliker, (SPIE Press, Bellingham, 2008), Chap 15, Vol. 6

${ }^{2}$ M. L. Calvo, P. Cheben, J. Opt. A: Pure Appl. Opt. 11, 024009 (2009).

${ }^{3}$ M. Haw, Nature 422, 556 (2003).

${ }^{4}$ D. Psaltis, F. Mok, Sci. Am. 273, 70 (1995)

${ }^{5}$ F. Mok, G. Zhou, D. Psaltis, Holographic Read-Only Memory in Holographic Data Storage, edited by H. J Coufal, D. Psaltis, G.T. Sincerbox, (Springer, Berlin, 2000), pp. 399-407.

${ }^{6}$ L. Solymar, D. J. Cooke, Volume Holography and Volume Gratings, (Academic Press, London, 1981), Chap 10.

${ }^{7}$ W. Wilson, A. Hoskins, M. Ayres, A. Hill, K. Curtis, Holographic Data Storage: From Theory to Practical Systems, edited by K. Curtis, L. Dhar, A. Hill, W. Wilson, M. Ayres, (John Wiley and Sons, Ltd, Chichester, 2010), Chap. 2.

${ }^{8}$ G. T. Sincerbox, Current Trends in Optics, edited by J. C. Dainty, (Academic, London, 1994), Chap 14, Vol. 2.

${ }^{9}$ R. A. Lessard, G. Manivannan, Proc SPIE 2405, 2 (1995).
${ }^{10}$ H. J. Coufal, D. Psaltis, G. T. Sincerbox, Holographic Data Storage (Springer, New York, 2000).

${ }^{11}$ P. Cheben, T. Belenguer, A. Nuñez, F. del Monte, D. Levy, Opt. Lett. 22, 1857 (1996).

${ }^{12}$ F. Del Monte, O. Martínez-Matos, J. A. Rodrigo, M. L. Calvo, P. Cheben, Adv. Mater. 18, 2014 (2006).

${ }^{13}$ P. Cheben, M. L. Calvo, Appl. Phys. Lett. 78, 1490 (2001).

${ }^{14}$ K. Omura, Y. Tomita, J. Appl. Phys. 107, 023107 (2010).

${ }^{15} \mathrm{P}$. Wassercheid, T. Welton, Ionic Liquids in Synthesis (Wiley, Weinheim, 2003).

${ }^{16}$ M. A. Klingshirn, S. K. Spear, J. D. Holbrey, R. D. Rogers, J. Mater. Chem. 15, 5174 (2005).

${ }^{17}$ A. J. Carmichael, D. M. Haddleton, S. A. F. Bon, K. R. Seddon, Chem. Commun. 14, 1237 (2000).

${ }^{18}$ K. Hong, H. Zhang, J. M. Mays, A. E. Visser, C. S. Brazel, J. H. Holbrey, W. M. Reichert, R. D. Rogers, Chem. Commun. 13, 1368 (2002).

${ }^{19}$ S. Harrisson, S. R. Mackenzie, D. M. Haddleton, Chem. Commun. 23, 2850 (2002)

${ }^{20}$ H. Lin, P. W. Oliveira, M. Veith, Appl. Phys. Lett. 93, 141101 (2008).

${ }^{21}$ H. Kogelnik, Bell. Syst. Tech. J. 48, 2909 (1969).

${ }^{22}$ H. Lin, P. W. Oliveira, M. Veith, M. Gros, I. Grobelsek, Opt. Lett. 34, 1150 (2009)

${ }^{23}$ M. L. Calvo, P. Cheben, O. Martínez-Matos, F. del Monte, J. A. Rodrigo, Phys. Rev. Lett. 97, 084801 (2006).

${ }^{24}$ O. Martínez-Matos, M. L. Calvo, A. Rodrigo, Appl. Phys. Lett. 91, 14115 (2007).

${ }^{25}$ O. Martínez-Matos, J. A. Rodrigo, M. L. Calvo, V. Hevia-Martín, P. Cheben, Inf. Opt. 18, 21 (2009).

${ }^{26}$ O. Martínez-Matos, J. A. Rodrigo, M. P. Hernández-Garay, J. G. Izquierdo, R. Weigand, M. L. Calvo, P. Cheben, P. Vaveliuk, L. Bañares, Opt. Lett. 35, 652 (2010). 